\section{A Compact Field Emission SEM for Low Voltage Imaging}

\author{
Jim Rynne \\ Novelx, Inc., Lafayette, CA \\ rynne@novelx.com
}

\section{Introduction}

A disruptive innovation in how scanning electron microscopes (SEMs) are designed and built has produced a low cost, but highperformance compact field emission SEM. By leveraging silicon processing technologies, Novelx has miniaturized the core technology inside a SEM. This miniaturization enabled the design of an all-electrostatic electron beam column that when coupled with a thermal field emission (TFE) electron source is optimized for lowvoltage imaging and sub-10nm resolution. In addition, the $m y S E M^{\odot}$ has a quad-segmented microchannel plate (MCP) detector that has a topographic imaging mode that is capable of electron channeling contrast imaging (ECCI) at low imaging voltages. These capabilities were previously only available in high-end and much larger sized field emission SEMs outfitted with additional detectors. The Novelx mySEM show in Fig. 1 is now being used in material science and life science applications for characterizing a variety of energy sensitive nanomaterials, biomaterials and thin films.

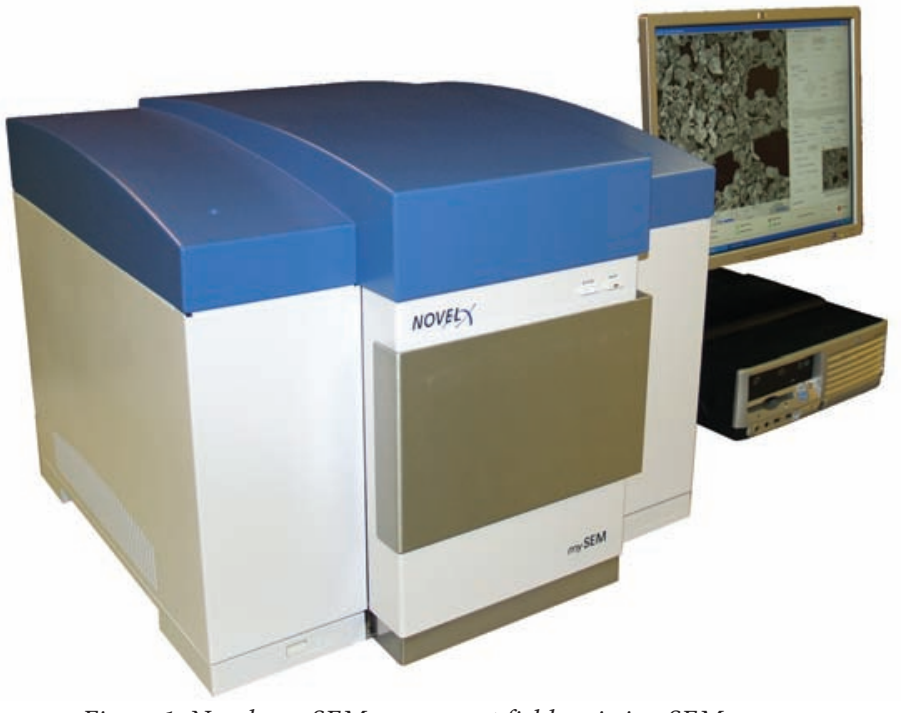

Figure 1: Novelx mySEM, a compact field emission SEM.

\section{Conventional Electron Beam Column Design}

The core technology inside a SEM is the electron beam column whose purpose is to extract, collimate, shape, scan, and focus the electron beam. A conventional electron beam column relies on a combination of precision-machined electromagnetic and electrostatic elements to control the electron beam. The coils that form the critical elements are typically hand wrapped individually by skilled technicians. This is needed to achieve the uniform electromagnetic fields required for high-resolution performance. At the system level, closed-loop cooling and sophisticated vibration isolation is generally required to manage the high currents in the lenses and other elements. The result is a high resolution electron beam column, but one that is expensive and large.

\section{A Miniature Electron Beam Column Design}

This same high-resolution performance can be realized at a significantly lower cost and in a smaller size with the miniaturization of an all-electrostatic electron beam column. A cross section and ray
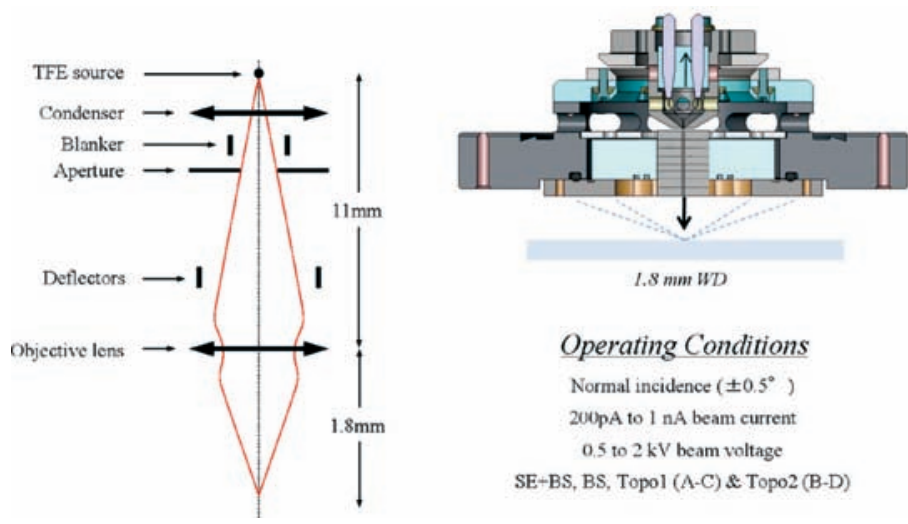

Figure 2: Novelx electron beam column design (left side) and mySEM FESEM cartridge implementation (right side) with operating conditions listed.

diagram of the complete FESEM cartridge is shown in Fig. 2.

Novelx uses stacks of silicon on insulator to form all the lens, apertures and deflectors needed in the electron beam column. This patented Stacked Silicon Technology ${ }^{\mathrm{Tx}}$ enables Novelx to build electron beam columns wafer-scale on $150 \mathrm{~mm}$ substrates. By leveraging semiconductor and bulk micromachining fabrication processes, Novelx fabricates columns with the precise aperture diameters and, moreover, the repeatable alignment tolerances that are needed to sufficiently minimize aberrations which can degrade image quality.

The source of electrons is the single biggest contributor to the fidelity and resolution of the images that a SEM can achieve. In order to ensure consistent, reliable performance, the Novelx mySEM was designed to use a TFE source. This source provides high brightness, high stability, small virtual source size, low energy spread and a long life of up to 10,000 hours of continuous operation. A quad-segmented MCP detector is located just below the objective lens of the electron beam column directly above the sample. This detector collects both backscattered and secondary electrons. The MCP may be operated either in a standard mode, where all the channels are added together, or in differential mode (topographic mode), in which opposite sides of the detector are dynamically subtracted. The electron source, the electron beam column and the detector are all combined into a field replaceable FESEM cartridge. When the electron source is depleted (typically after about 10,000 hours of continuous operation), the entire FESEM cartridge can be replaced in the field to provide the mySEM with not only a new source of electrons, but a new pre-aligned electron beam column.

\section{High Resolution Imaging}

Most SEMs, and in particular most compact SEMs, quote imaging performance at a high accelerating voltage. High voltage imaging is problematic because the higher energy electrons can damage samples and cause non-conductive or biological samples to charge, making them difficult to image. Also, as the accelerating voltage is increased, the electron penetration depth increases thereby reducing the resolution at the sample surface. For some systems only a magnification specification is provided or when a resolution specification is provided, the imaging voltage is not. Magnification alone, however, is not a good indication of a systems performance since there is not a standard definition for $1 \times$ magnification. For this reason, Novelx quotes resolution at $1 \mathrm{keV}$. High resolution at a low accelerating voltage is more difficult to achieve and has previously been available only in high-end systems. Every mySEM is guaranteed to achieve $<10 \mathrm{~nm}$ resolution at $1 \mathrm{keV}$. There are several methods to determine resolution, the simplest and most common being to demonstrate feature 



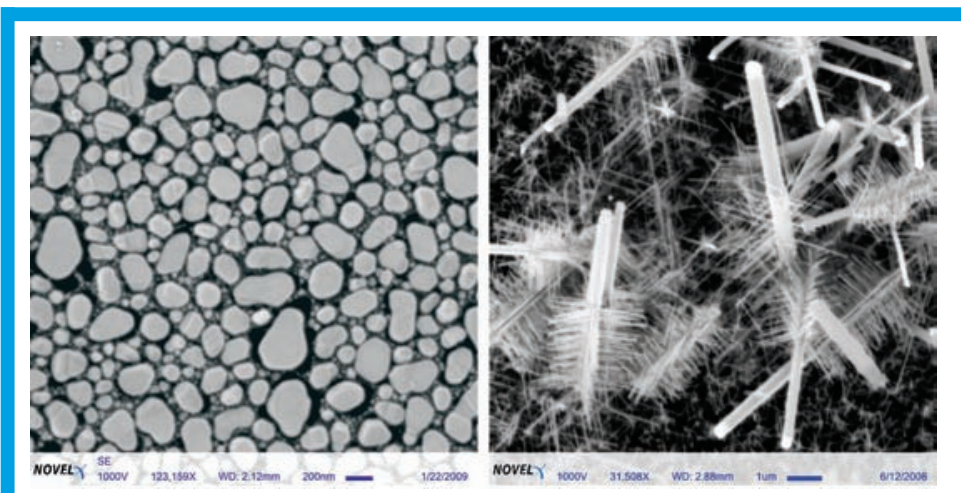

Figure 3: The image on the left side shows as 20nm Au islands on Carbon separated by gaps as small as $5 \mathrm{~nm}$. The image of branching $\mathrm{Si}$ Nanowires on the right side is provided courtesy of UC Berkeley COINS.

size and separation on a calibration sample. Figure 3 shows a mySEM image of good calibration sample, gold islands on carbon. The sample has gold islands as small as $20 \mathrm{~nm}$ with gaps between the islands as small as $5 \mathrm{~nm}$. Novelx always uses both calibration samples and user samples to demonstrate performance and ensure that the mySEM can resolve the features important to a user's application. The growth and study of branching Silicon nanowires is one such application requiring high resolution imaging [1]. The Novelx mySEM image of a "forest of nanotrees" shown in Fig. 3 is provided courtesy of the UC Berkeley Center of Integrated Nanomechanical Systems (COINS). The image shows nanowire branches 25 to $60 \mathrm{~nm}$ in diameter.

\section{Low Voltage Imaging Techniques}

Several imaging techniques are available with the Novelx $m y$ SEM to enhance surface contrast and allow nanoscale features to be observed. A variable low-voltage system eliminates sample charging of non-conductive samples without the need to coat samples, which can mask many nanoscale features, or to operate at increased pressure, which can degrade resolution. By adjusting the beam voltage to the stable crossover point on the material's electron yield curve, sample damage and charging can be minimized while providing increased contrast and surface detail. The mySEM images in Fig. 4 are of an uncoated Schistosoma mansoni parasite (images provided courtesy of the UCSF Sandler Center for Basic Research in Parasitic Diseases).
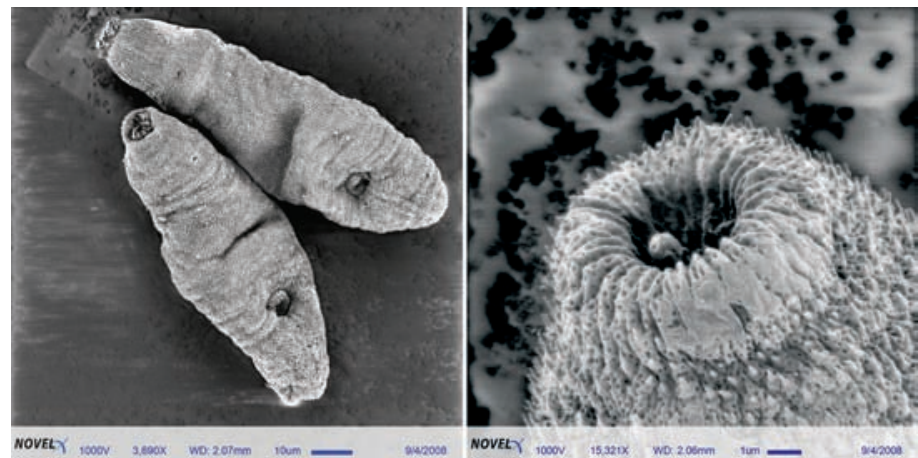

Figure 4: Images of uncoated Schistosoma mansoni parasites courtesy of the UCSF Sandler Institute for Basic Research in Parasitic Diseases.

\section{Topographic Imaging Techniques}

The mySEM may be operated in either a standard mode, where all the channels of the MCP detector are added together, or in a topographic mode, in which opposite sides of the detector are dynamically subtracted. The topographic effect is similar to positioning a light source in optical microscopy at a glancing incidence. Any combination of the segments may be used for imaging [2]. An example of a topographic mode image of a $20 \mathrm{~nm}$ thick holey carbon sample and a comparison to a standard mode image is shown in Fig. 5.
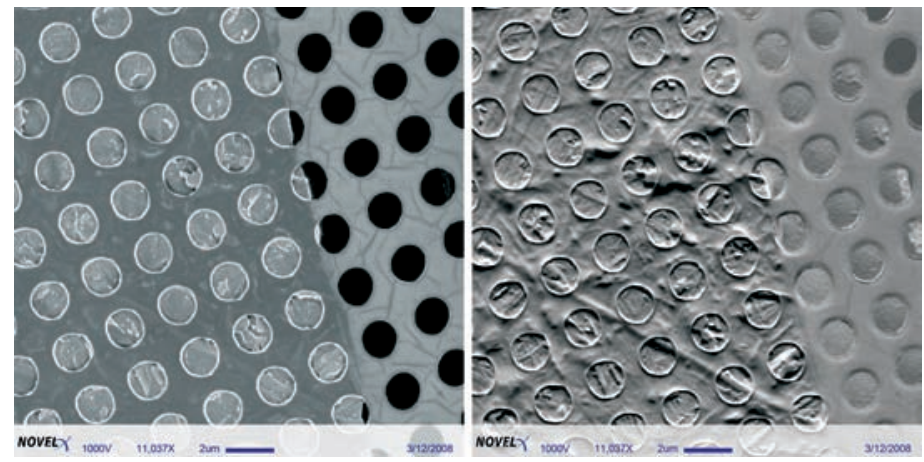

Figure 5: Comparison of standard mode (left side) to topographic mode (right side). The sample consists of a $20 \mathrm{~nm}$ thick carbon film on a standard copper TEM grid. The holes are $1.2 \mu \mathrm{m}$ in diameter on a $2.5 \mu \mathrm{m}$ pitch.

\section{Electron Channeling Contrast Imaging (ECCI) Technique}

To explore the resolution limits of the mySEM's topographic mode, single crystal $6 \mathrm{H}$-SiC wafers were imaged [2]. The wafers were known to have terraced structures of varying heights. The images shown in Fig. 6 were taken with the mySEM in topographic mode and are consistent with electron channeling contrast imaging (EECI) at low voltages.

The images (provided courtesy of UC Berkeley COINS) show hillocks, defects and terraced planes on the sample. The fine structure on the terraces was determined and independently verified with an atomic force microscope to be sub-nm steps of $\sim 0.8 \mathrm{~nm}[2]$. The unique geometry of the mySEM's miniature electron beam column coupled with its high brightness TFE and MCP detector, all contributed to the mySEM being able to image this silicon carbide sample and possibly other crystalline substances using electron channeling techniques.

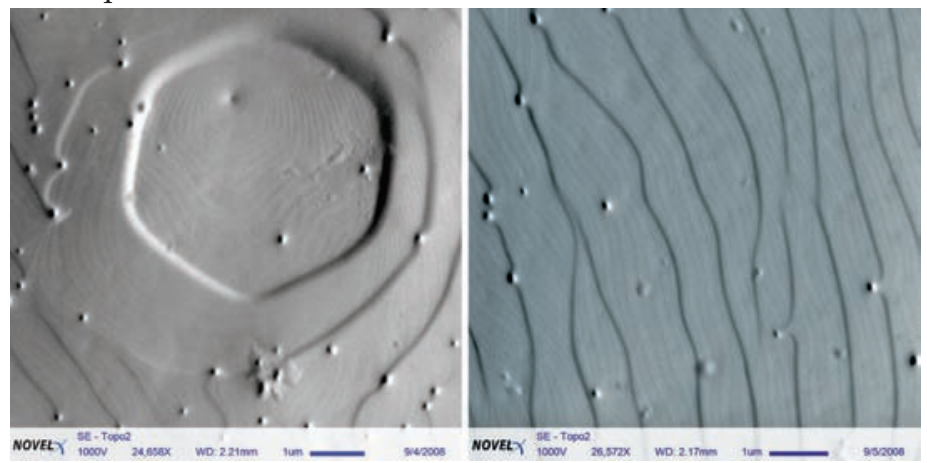

Figure 6: The topographic mode image of the $6 \mathrm{H}-\mathrm{SiC}$ sample on the left side shows several surface features on the sample. The image on the right side shows fine structure on the terraces.

\section{Conclusion}

The Novelx mySEM is a compact field emission SEM that offers capabilities previously only available in more expensive and much larger conventional field emission SEMs. The patented Stacked Silicon Technology and miniature electron beam column design of the mySEM provides sub-10nm resolution and several low-voltage imaging techniques that should prove valuable in a wide variety of material science and life science applications.

\section{References}

[1] G. S. Doerk, N. Ferralis, C. Carraro and R. Maboudian, "Growth of branching Si nanowires seeded by Au-Si surface migration." Journal of Materials Chemistry (7/2008).

[2] L. P. Muray, J. P. Spallas, C. S. Silver, S. Indermuehle, N.Ferralis, C. Carraro, and R. Maboudian, "Resolving sub-nanometer steps with a low voltage miniature scanning electron microscope," Microelectronic Engineering (1/2009). 


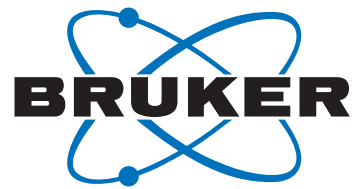

\section{EDS for nanoanalysis}
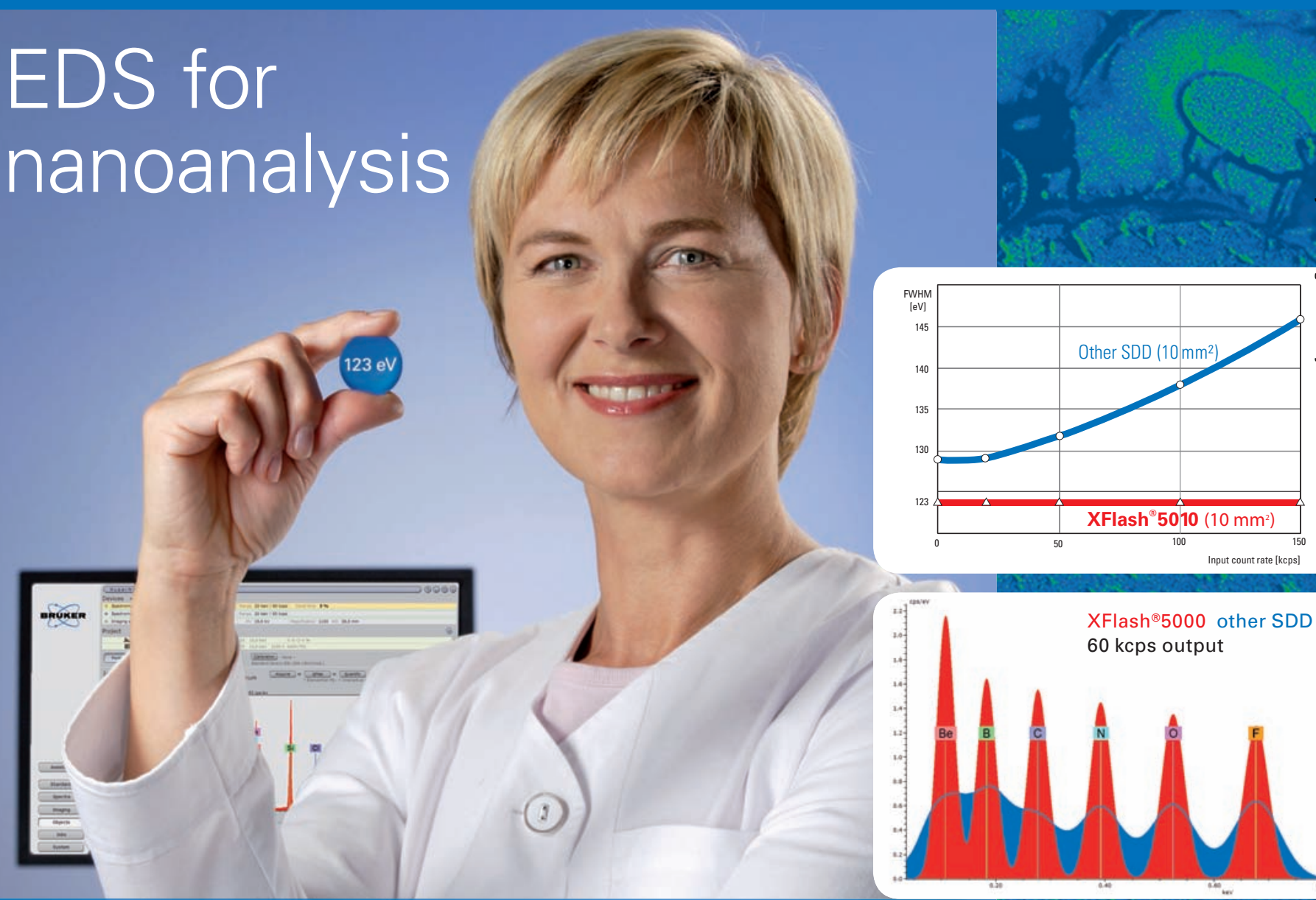

\section{XFlash ${ }^{\circledR} 5000$ - best resolution ever! High collecting efficiency and $123 \mathrm{eV}$ even at $100,000 \mathrm{cps}$}

The XFlash ${ }^{\circledR}$ silicon drift detectors are part of our QUANTAX EDS systems

- Designed for high resolution FE-SEMs

- Superb light element performance (C Ka $\leq 46 \mathrm{eV}, \mathrm{F} \mathrm{K \alpha} \leq 54 \mathrm{eV})$

- Detection from beryllium (4) to americium (95)

- Worldwide most comprehensive atomic data library now includes $\mathrm{N}$-lines

- $L N_{2}$, vibration and maintenance free

- XFlash ${ }^{\circledR}$ SDDs are available with 10,30 and $40 \mathrm{~mm}^{2}$ active area

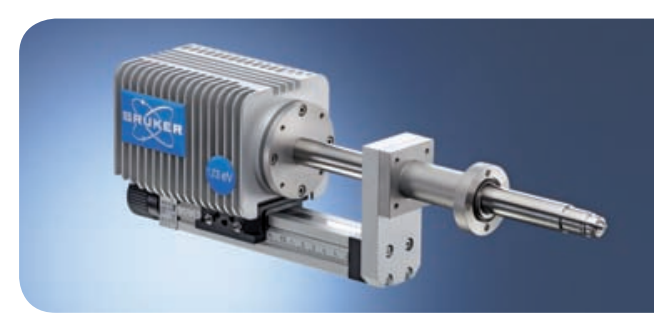

www.bruker-axs-microanalysis.com 